\title{
AgNPs-based Label-Free Colloidal SERS Nanosensor for the Rapid and Sensitive Detection of Stress-Proteins Expressed in Response to Environmental-Toxins
}

\author{
Vinay Bhardwaj, Supriya Srinivasan and Anthony J McGoron* \\ Department of Biomedical Engineering, Florida International University, 10555 West Flagler Street, Miami, USA
}

\begin{abstract}
Among several physical, chemical and immunoassay-based methods for the detection of biomolecules, the Enzyme-Linked Immuno-Sorbent Assay (ELISA) is the standard technique that is routinely used for quantification of known proteins. However, it is a label-based, end-point sensor technique that is time-consuming, labor-intensive and fairly costly. This sandwich assay typically involves a series of peptide binding and washing steps. Here, we report a Surface-Enhanced Raman Spectroscopy (SERS) immuno-nanosensor technique that allows rapid and label-free extracellular detection of proteins compared to ELISA, and can potentially be used for intracellular detection. Our study shows that the silver nanoparticles (AgNPs) based SERS sensor can detect the stress-proteins, HSP70 and RAD54 expressed by yeast in response to environmental-toxins, in a dose dependent manner. As compared to the multi-step sandwich ELISA technique, the detection of stress-proteins using the SERS sensor is a simple two-step process. The simplicity of the SERS nanosensor design allowed the rapid detection of proteins within two hours in a fairly cost-effective and user-friendly approach. The SERS sensor we reported has an edge over ELISA as it directly quantifies the proteins without using any label (label-free) and also gives qualitative information about the antigen-antibody interaction. The SERS sensor showed good correlation and comparable sensitivity with ELISA. However, SERS was found to be less reproducible. Compared to previous reports on SERS-based protein detection techniques, our colloidal SERS sensor is easy to fabricate, offers improved biocompatibility, and allows rapid detection of the proteins in a cellular environment at picogram-levels. As a result, the SERS sensor demonstrates great potential for biomedical and environmental sensor technology (BEST) allowing label-free, rapid and sensitive detection, and could possibly replace ELISA.
\end{abstract}

Keywords: Enzyme-linked immuno-sorbent assay (ELISA); Surfaceenhanced Raman spectroscopy (SERS); Label-free, Silver nanoparticles (AgNPs); Stress-proteins; environmental-toxins

\section{Introduction}

There is an increasing threat of deliberate or accidental contamination of the environment with Warfare Agents (WAs); chemical and biological, Toxic Industrial Chemicals (TICs), and other types of toxins. There is a need for the development of a "detect to protect" portable environmental biosensor that can rapidly detect, identify and monitor these toxins at sub-lethal levels. Almost all the current biosensor technologies employed for the detection of environmental-toxins can be categorized on the basis of three properties: (i) label-based or label-free detection (ii) extracellular or intracellular detection and (iii) portability. Most of the biosensor technologies, such as enzyme-linked immuno-sorbent assay (ELISA), Fluorescence Resonance Energy Transfer (FRET) and bioreporter employing fluorophore dyes (labels) are label-based sensors [1-3]. Toxicity, photo bleaching, customized synthesis and conjugation are a few of the numerous limitations of the use of fluorophores [4]. Label-based sensors introduce uncertainty in measurements as they indirectly determine the concentration of analytes through the signal obtained from the label-analyte conjugate. Enzyme-Linked ImmuneSorbent Assay (ELISA) is the standard technique accepted widely for the detection of proteins. However, it is a label-based detection from extract obtained by lysing the cells after a definite time period (endpoint sensor). ELISA involves a series of peptide (antigen-antibody) binding and washing steps that make it time consuming, labor intensive, and also add to the cost. Whole-Cell Biosensor Technologies (WCBT) that do not use any label (label-free) have been developed [5]. Label-free WCBT, such as Surface Plasmon Resonance (SPR), Quartz Crystal Microbalance (QCM), Ion-Selective Field Effect Transistor (ISFET), Electric Cell-Substrate Impedance Sensing (ECIS) and a few others are highly sensitive and rapid. However, their non-specificity is the key drawback [6]. Almost all of the early works related to WCBT are centered on cell-adhesion [5] and lack intracellular detection. Whole-cell living biosensor technology using engineered cells and microbes (bioreporters) have been developed for real-time intracellular detection. However, this technology has not yet enjoyed commercial success due to several constraints, including the use of Genetically Modified Organisms (GMOs) [7]. Bioreporters for intracellular detection of stress-proteins such as HSP70 and RAD54 have emerged as a successful portable sensor technique for environmental applications $[8,9]$. The high incubation time of the assay ( 1 day) further limits their application to risk-management of contaminated sites and fail as a rapid "detect to protect" sensor device. As of now, none of the existing environmental sensor technologies is able to replace ELISA and satisfy the most demanding requirements: label-free, portable, dynamic, and allow intracellular detection.

The most obvious extension to current successful optical sensor technology is Surface-Enhanced Raman Scattering (SERS). The

*Corresponding author: Anthony $\mathrm{J}$ McGoron, Department of Biomedical Engineering, Florida International University, 10555 West Flagler Street, Miami, USA, Tel: 305-348-1352; E-mail: mcgorona@fiu.edu

Received October 21, 2013; Accepted November 18, 2013; Published November 25, 2013

Citation: Bhardwaj V, Srinivasan S, McGoron AJ (2013) AgNPs-Based LabelFree Colloidal SERS Nanosensor for the Rapid and Sensitive Detection of StressProteins Expressed in Response to Environmental-Toxins. J Biosens Bioelectron S12: 005. doi: 10.4172/2155-6210.S12-005

Copyright: @ 2013 Bhardwaj V, et al. This is an open-access article distributed under the terms of the Creative Commons Attribution License, which permits unrestricted use, distribution, and reproduction in any medium, provided the original author and source are credited. 
enhancement of Raman scattering occurs when metal Nanoparticles (NPs) such as gold $(\mathrm{Au})$ and silver $(\mathrm{Ag})$ are in close proximity to an analyte [10]. Au and Ag have their own advantages and disadvantages, such as Au is more biocompatible but less SERS active than Ag. Owing to the highly sensitive and label-free detection based on the chemical signature of the analyte, SERS is used for characterization and detection of biomolecules [11], particularly proteins [12]. The induction of proteins in response to stress (stress-proteins) is well characterized in yeast, and yeast holds numerous advantages over bacterial and mammalian cells to fabricate portable biosensors [13]. The stress response of yeast and their homology with higher eukaryotes is well characterized [14].

In the event of release of toxins into the environment, including WAs and TICs, their rapid detection and identification at sub-lethal levels is required. For known or specified toxic agents, an assay can be readily developed. Indeed, many sensors already exist. However, there is an acute need for the development of a non-specific sensor that can respond to the release of unknown toxicants. Such a sensor needs to be rapid, user-friendly and cost-effective so it can be used by the military and civilians during the release of any suspected toxicants. The standard cytogenetic methods and ELISA technique fail to meet these requirements. A Raman spectroscopy cell-based biosensor has been reported to monitor warfare agents such as ricin and sulphur mustard [15]. However, there is no elucidation of stress-protein expression in response to environmental-toxins using SERS. In this article we demonstrate the progress of a SERS immunosensor technology that can possibly be used as a rapid, user-friendly and inexpensive sensor for detection of the biological response to environmental toxins, potentially replacing ELISA. The technique was validated by the dose dependent extracellular detection of two stress proteins, HSP70 and RAD54, expressed by yeast in response to two common environmentaltoxins, $\mathrm{UV}$ and $\mathrm{H}_{2} \mathrm{O}_{2}$.

\section{Experimental}

\section{Preparation of SERS substrate and nanosensor}

Silver colloidal Nanoparticles (NPs) were prepared by the single step citrate reduction process [16] with some modifications. The modifications were introduced to minimize the transformation of the AgNPs into ionic silver, which can impact their stability and biocompatibility [17]. All glass apparatus used in the preparation of the SERS substrate (colloidal AgNPs) were washed with aqua regia $(\mathrm{HCl}$ and $\mathrm{HNO}_{3}$ in 3:1). Ultrapure deionized molecular biology grade water was used for preparing solutions. The SERS potential of the AgNPs was evaluated using Rhodamine-6-G (R-6-G) as a probe molecule and the average SERS Enhancement Factor (EF) was calculated at a single characteristic peak [18]. Considering the three-fold SERS potential of AgNPs over AuNPs of similar morphology (data not shown) the Ag based substrate was chosen for the fabrication of SERS nanosensors.

The anti-HSP70 and anti-RAD54 Monoclonal Antibodies (MAbs) were conjugated to the Ag substrate through the bifunctional linker Mercapto-Methyl-Thiazoleacetic acid (MMT) following carbodiimide chemistry [19]. The protocol was modified to increase surface coverage of NPs by MAbs and decrease agglomeration of the sensor. Briefly $5 \mu \mathrm{l}$ of $50 \mathrm{mM}$ MMT solution dissolved in 15\% ethanol was added to each $\mathrm{ml}$. of AgNPs $\left(\sim 7 \times 10^{10} \mathrm{NPs} / \mathrm{ml}\right)$ and incubated for 2 hours at room temperature under mild mixing conditions. The linker-NP solution was thoroughly washed and the free carboxylic groups on the linker were activated by carbodiimide chemistry (ethyl dimethylaminopropyl carbodiimide (EDC)/ N-hydroxysuccinimide (NHS) in $2.8 / 1.2 \mathrm{mM}$ ).
The reaction time was 2 hours at $20^{\circ} \mathrm{C} .100 \mu \mathrm{l}$ of $2 \%$ bovine serum albumin (BSA, purchased from Sigma Aldrich U.S.A. product\#A7030) was added to cover the remaining surface of the sensor to avoid any non-specific binding of the molecules to the sensor. The sensor was thoroughly washed and resuspended at $10 \mu \mathrm{g} / \mathrm{ml}$ concentration before its physical and chemical characterization was performed. The physical properties including size, size distribution, zeta potential (surface charge), shape and concentration of the substrate and the sensor were measured using a Cary Varian UV-Vis spectrophotometer from Agilent Technologies, zetasizer NanoZS from Malvern Instruments Ltd., CM200 Transmission Electron Microscope (TEM) from Philips and inductively coupled plasma mass spectrometer (ICP-MS) from PerkinElmer. The chemical composition was confirmed using a Raman spectro-microscope from PerkinElmer. The response of the SERS sensor was evaluated by measuring the standard proteins in pure solution and in a mixture with R-6-G to test the sensitivity and selectivity of the sensor. The selectivity of the sensor was also tested using yeast extract simulating the cellular environment of yeast. All the chemicals and reagents were purchased from Sigma Aldrich (U.S.A.) except the peptides, which were purchased from the manufacturers of the ELISA kits.

\section{Exposure of yeast to stress-toxins and detection of stress- proteins using ELISA and SERS}

A mixed culture of Baker's yeast Saccharomyces cerevisiae (S.c.) and a pure culture of S.c. (BY4742) were used in the study. Both cultures were grown in Yeast Extract-Peptone-Dextrose (YPD) broth at optimum conditions, $30^{\circ} \mathrm{C}$ for 3 days in an incubator shaker. Cell density was measured indirectly using the Beer Lambert's equation with absorption at $600 \mathrm{~nm}$ (optical density) and directly by counting cells with a haemocytometer, as well as by directly counting colonies on the plates. The effect of silver on yeast growth was studied previously [20]. Briefly, $2.5 \mathrm{ml}$ of culture media was inoculated with 30,000 cells of mixed and pure culture separately at time zero $\left(\mathrm{t}_{0}\right)$. The AgNPs (SERS substrate), polymer (MAbs and BSA) coated AgNPs (SERS sensor) and ionic silver (silver nitrate salt) was added to the inoculated culture at a final concentration of $10 \mu \mathrm{g} / \mathrm{ml}$ and incubated for 12 hours $\left(t_{12}\right)$. Cells without any test agent exposure were considered as control.

The mixed culture, which has high resistance to $\mathrm{Ag}$, was grown under optimum conditions and the cells were harvested at log phase $\left(10^{7}\right.$ cells $/ \mathrm{ml}$ ). One $\mathrm{ml}$ of yeast culture was exposed to environmental stresstoxins $\left(\mathrm{H}_{2} \mathrm{O}_{2}: 5,50,500 \mathrm{mM}\right.$ and U.V.: A, B, C i.e. 365,302 and $\left.254 \mathrm{~nm}\right)$ for 15 minutes and 60 minutes, respectively. Control (unexposed) and treated (exposed) cells were lysed using the yeast-protein extraction reagent (Y-PER) from Thermo-Scientific, following the manufacturer's protocol. The yield of total extracted proteins was measured using a commercial Bicinchoninic Acid (BCA) kit. The specific proteins, HSP70 and RAD54, were measured using commercial ELISA kits and the SERS nanosensors we fabricated. The correlation in accuracy and the efficiency of the two sensors in the extracellular detection of proteins was compared. The sensitivity of the two sensors was compared in terms of Lowest Limit of Detection (LLD) and the Linear Range of Detection (LRD). The LLD was the lowest concentration of proteins differentiated from a blank sample. The LRD and the reproducibility of the sensors were determined by plotting calibration curves (intensity vs. amount) of the pure proteins in solution to find the correlation coefficient $\left(\mathrm{r}^{2}\right)[21]$.

\section{SERS instrumentation and measurement}

The PerkinElmer Raman spectroscope (Raman Station 400F) and 
microscope (Raman Micro 300), which offer an easy to use macromicro sampling system with permanently aligned optics, were used to acquire the SERS spectra. The system uses a diode laser operating at 785 $\mathrm{nm}$ with average power of $100 \mathrm{~mW}$ at the sample and 100-micron spot size. Inbuilt spectrum software was used for processing the spectra, such as baseline correction, normalization, background subtraction, peak assignment and resolution. All spectra were acquired using a laser exposure time of 5-15 seconds (5 acquisitions of 1-3 seconds each). For low sample volumes and chemical characterization of the SERS sensor the Raman microscope was used. A drop of sample was placed on a pre-cleaned glass slide and manually focused under a $20 \mathrm{X}$ objective, scanning the sample from the center to the edge. The Raman spectroscope was used for High Throughput Screening (HTS) of the stress-proteins. The $200 \mu \mathrm{l}$ of sample volume loaded in a glass-bottom 96 well plate was scanned using mapping mode (step size: $100 \mu \mathrm{m}$ ). Samples, loaded in a glass bottom well-plate in duplicate, were analyzed at three different spots per sample $(2 \times 3$ spectra for each sample) to get the mean intensity of the Raman peaks. The experiment was repeated three times $(n=3)$. The intra and inter batch deviation in intensity of the Raman peaks was reported as mean \pm S.D. to assess reproducibility [22].

\section{Results and Discussion}

\section{Evaluation of SERS substrate}

Almost spherical silver colloidal NPs of about $60 \mathrm{~nm}$ size were prepared. Figure 1 shows the morphological characterization of the AgNPs-based SERS substrate. The characteristic color and the absorption peak indicate the formation of AgNPs. The NPs have narrow size distribution (polydispersity index 0.14 ) as observed by the narrow absorption peak and the DLS histogram. The substrate was highly negatively charged $(-40 \mathrm{mV})$ due to citrate groups. The citrate is the most commonly used carboxylic acid that acts as reducing as well as capping-agent (coating) for the AgNPs. The citrate-cap holds two major significances. First; the oxo $\left(\mathrm{O}^{-}\right)$and hydroxo $\left(\mathrm{OH}^{-}\right)$groups of the citrate offer high repulsive forces that provide stability to the NPs. Second; these groups are easily replaced by other more reactive functional groups, such as thiol ( $\mathrm{SH})$ thereby allowing facile conjugation of biomolecules to the Au and AgNPs. The concentration of the AgNPs estimated using ICP-MS was $100 \mathrm{ppm}(0.1 \mathrm{mg} / \mathrm{ml})$, roughly equivalent to $7 \times 10^{10} \mathrm{NPs} /$ $\mathrm{ml}$. The shape, size, charge and concentration of the AgNPs are in good agreement with other groups that used a similar citrate reduction process $[23,24]$.

The average SERS Enhancement Factor (EF) of the AgNPs was calculated using R-6-G as probe molecule and the simple equation given by:

$$
\mathrm{EF}=\left(\mathrm{N}_{\mathrm{vol}} / \mathrm{I}_{\text {surf }}\right) \cdot\left(\mathrm{N}_{\text {surf }} / \mathrm{I}_{\text {vol }}\right)
$$

$\mathrm{N}_{\mathrm{vol}}$ and $\mathrm{N}_{\text {surf }}$ stand for the numbers of probe molecules in aqueous sample volume and on the surface of SERS substrate, respectively. $\mathrm{I}_{\mathrm{vol}}$ and $\mathrm{I}_{\text {surf }}$ are the corresponding Raman and SERS intensities. The prominent peak of R-6-G at $1503 \mathrm{~cm}^{-1}$ and the footprint area $20 \AA^{2}$ was considered for the calculation of EF. The $\mathrm{N}_{\text {surf }}$ is estimated to be $4.87 \times 10^{11}$ and the Nvol to be $2.47 \times 10^{14}$. The ratio of $I_{\text {surf }}$ to $I_{\text {vol }}$ for AgNPs is estimated to be $8.92 \times 10^{2}$ and therefore the $\mathrm{EF}$ is calculated as $4.52 \times 10^{5}$. Gold nanoparticles (AuNPs) of similar morphology under similar conditions showed little SERS potential ( 3 fold less than AgNPs) (data not shown). Therefore, Ag substrate was used for fabrication of the SERS nanosensors. Theoretical calculations indicate that single $\mathrm{Au}$ and $\mathrm{Ag}$ NPs can reach the maximum EF of $10^{3}-10^{4}$ and $10^{6}-10^{7}$, respectively [25]. The EF of our colloidal AgNPs is comparable to 3D silver shells [18], which is an improvement (almost 1 fold) over other conventional geometries, such as colloids and films [22]. The higher laser power at the sample $(100 \mathrm{~mW})$ compared to other groups possibly resulted in such a high EF from the colloidal AgNPs we prepared. The enhancement by the SERS substrate can further be increased by patterning substrates in a highly ordered arrangement (array) or using aggregating agents [26]. However, both strategies limit the wider applications of SERS sensors as compared to a colloidal sensor, particularly for intracellular SERS applications. The SERS-enabled nano-pipette technique based on microinjection has been demonstrated for intracellular detection [27]. However, the poor selectivity, low throughput and cellular manipulation that could lead to physical-stress limit this approach for our application.

\section{Evaluation of SERS nanosensor}

Considering the high SERS potential of AgNPs, they were used for the fabrication of the SERS sensor for the detection of stress-proteins. The size of the AgNPs after conjugation of anti-stress MAbs increased to $98 \mathrm{~nm} \pm 12 \mathrm{~nm}$ and surface charge increased to $-18 \mathrm{mV} \pm 7 \mathrm{mV}$, as confirmed by TEM, DLS and zeta analysis. Figure 2 shows the chemical characterization of the RAD54 SERS sensor at each step of the sensor fabrication: a) preparation of AgNPs, b) conjugation of MMT crosslinker to AgNPs (AgNPs-MMT) and c) conjugation of MAbs to AgNPs-MMT solution (SERS sensor).

The AgNPs prepared using the simple process involving just two chemicals, silver nitrate salt and the sodium citrate, have low Raman signals. The noticeable bands around $1060 \mathrm{~cm}^{-1}$ and $245 \mathrm{~cm}^{-1}$ are assigned to nitrate $\left(\mathrm{NO}^{3-}\right)$ stretching and are used to monitor the conjugation process. The successive decrease in this band confirms the replacement of nitrate groups by the functional groups of linker and the MAbs. The nitrate band has been used for characterization of AgNPs and as an internal standard during immuno-sensor fabrication
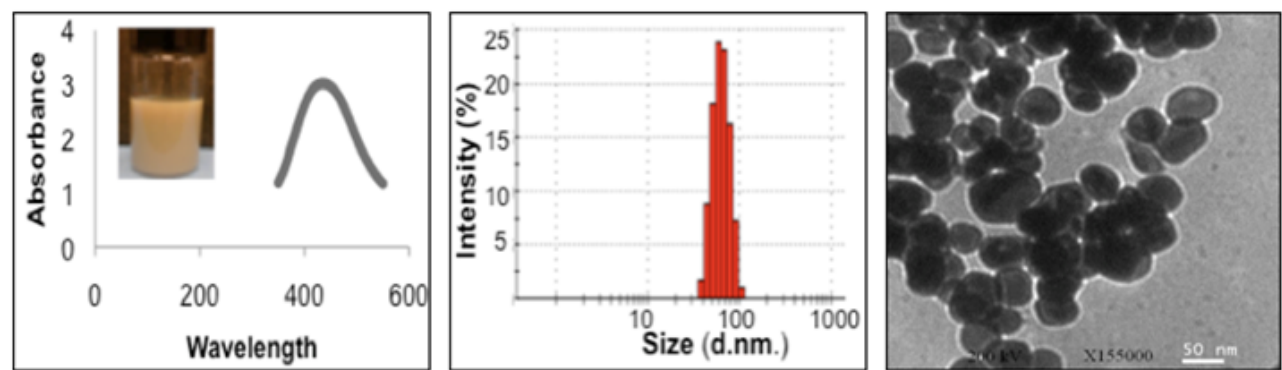

Figure 1: Characterization of colloidal AgNPs (SERS substrate). Characteristic yellow-greenish color (inset) and absorption peak at $436 \mathrm{~nm}$ (left) indicates the formation of AgNPs. DLS histogram (middle) and TEM image (right) further validates the AgNPs are almost spherical with $\sim 60 \mathrm{~nm}$ size. 


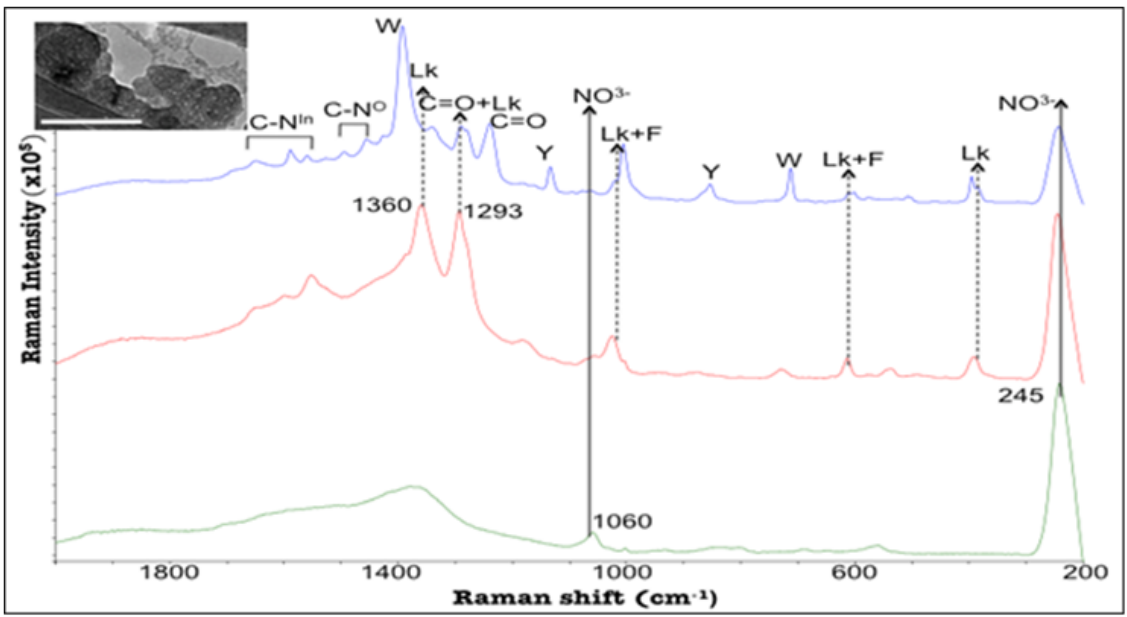

Figure 2: Chemical characterization of the RAD54 SERS sensor. Anti-RAD54 MAbs conjugated to AgNPs via MMT linker (RAD54 SERS sensor) shows the weak peaks characteristic to AgNPs (Bottom) and MMT linker (Middle) and strong peaks associated with protein or MAbs (Top). The solid and the dotted arrows represent decrease in characteristic peaks of AgNPs (NO ${ }^{3-}$ ) and the MMT linker (Lk), respectively. Protein bands are assigned to aromatic amino acids (W: Tryptophan, Y: Tyrosine and F: Phenylalanine) and amide bonds (I: C=O, II: C- $\mathrm{N}^{\circ}, I I I: C-\mathrm{N}^{\mathrm{ln}}$ where O is out of phase and In for in-phase). The inset TEM image (scale bar: $0.2 \mu \mathrm{m}$ ) of the RAD54 SERS sensor shows the MAbs conjugated to AgNPs.
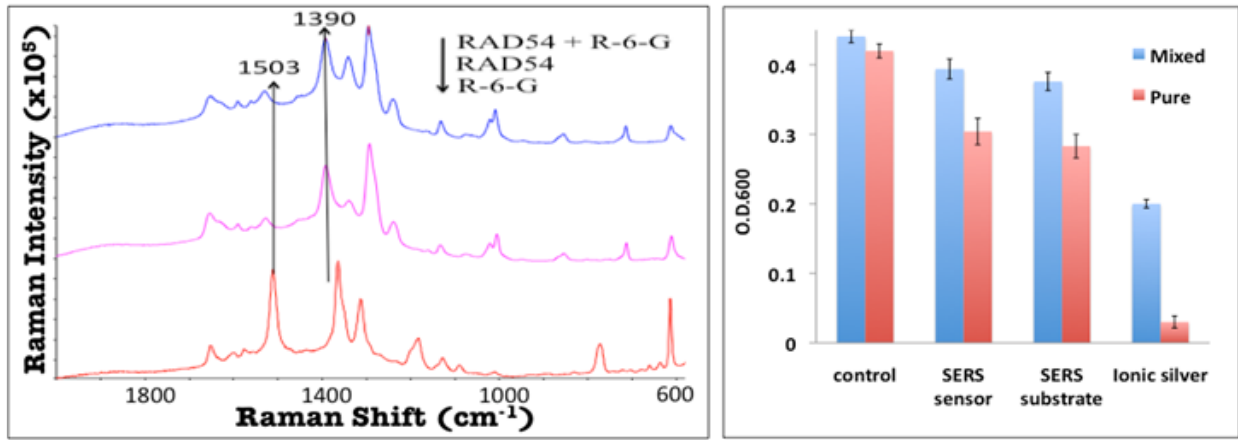

Figure 3: Selectivity (left) and toxicity (right) of the RAD54 SERS sensor. (Left) RAD54 protein in a mixture with R-6-G detected using the RAD54 sensor (top spectra) do not show the characteristic distinct peak of R-6-G at $1503 \mathrm{~cm}^{-1}$ (bottom spectra) but only the RAD54 distinct peak at $1390 \mathrm{~cm}^{-1}$ (middle spectra). (Right) The SERS sensor has less anti-microbial activity than the bare SERS substrate and ionic silver (Ag ${ }^{+}$). The pure BY4742 S.c. is more sensitive to growth inhibitory effect of $\mathrm{Ag}$ than to the mixed S.c. culture. The optical density (O.D.) of the inoculum was 0.15 . The error bars represent the S.D. of $n=3 \times 2$ (three independent experiments done in duplicate).

$[28,29]$. The decrease in intensity of the nitrate peaks and the addition of two main bands around $1300 \mathrm{~cm}^{-1}$ characteristic to the MMT linker have been reported [19]. Three Raman active aromatic amino acids: tryptophan $(\mathrm{W})$, phenylalanine $(\mathrm{F})$ and tyrosine $(\mathrm{Y})$ present in high amounts in both proteins (MAbs) contributed to the Raman signals, with peaks around 1390, $712(\mathrm{~W}), 1005,600(\mathrm{~F})$ and 1133, $853(\mathrm{Y})$. The successful conjugation of MAbs to the linker via amide bonds (CO$\mathrm{NH})$ is validated by the weak peaks originating from amide $\mathrm{I}(\mathrm{C}=\mathrm{O}$ around 1240 and 1290), amide II (out of phase C-N stretching around 1454 and 1494) and amide III (in phase C-N stretches between 1560 to 1660). The Raman peaks of the proteins are in good agreement with the literature [30,31]. The inset in Figure 2 shows the TEM image of the SERS sensor.

BSA has traditionally been used as a blocking agent to avoid Non-Specific Binding (NSB). However, all BSAs are not alike in their preparation and therefore differ in their NSB resistance [32]. The crude BSA and other ionic blocking-agents, such as casein, gelatine and dry milk, have high globulin content and fatty acids, which have high affinity to biomolecules circulating in a real environment [33]. Therefore, we used Fatty-Acid-Free and Globulin-Free (FAFGF) preparation of BSA
(Sigma\#A7030). The response of the sensor coated with FAFGF-BSA (blocking agent) was evaluated by detecting the stress-proteins in a mixture with the Raman active dye R-6-G to test the selectivity of the sensor (Figure 3 left). SERS gives rich information about the analytes and often results in their spectral overlapping. Therefore, the distinct R-6-G $\left(1503 \mathrm{~cm}^{-1}\right)$ and protein $\left(1390 \mathrm{~cm}^{-1}\right)$ peaks were considered for all qualitative (selectivity testing) and quantitative (calculation of $\mathrm{EF}$ and sensitivity) studies. The selectivity of the sensor was also tested in the presence of yeast extract to simulate the cellular environment of the yeast. Similar positive results were obtained (data not reported).

There are numerous reports on the huge variation in toxicity values associated with AgNPs, perhaps arising from differences in their preparation and testing environments [34]. We tested our SERS sensor for its growth inhibition effect to yeast. The toxicity of MAb conjugated and BSA coated AgNPs (SERS sensor), bare or citrate-capped AgNPs (SERS substrate) and ionic $\mathrm{Ag}\left(\mathrm{Ag}^{+}\right.$from silver nitrate solution) were tested on two types of yeast S.c. culture: mixed and pure BY4742. Figure 3 (right) shows the growth inhibition effect of the test agents on the mixed culture at the SERS effective dose $(10 \mu \mathrm{g} / \mathrm{ml}$ roughly equivalent to $10^{10} \mathrm{NPs} / \mathrm{ml}$ ) and time (12 hours). The cellular uptake of colloidal 
metal NPs has been reported previously to reach saturation at the chosen dose and time [35].

The bare AgNPs-based SERS substrate and the sensor both have improved biocompatibility over what other groups have reported [34]. The improved biocompatibility is perhaps because the transformation of AgNPs (oxidation and dissolution to $\mathrm{Ag}^{+}$and the agglomeration) that impacts toxicity was controlled by designing our experiment in reference to a critical review [17]. Different physical and chemical parameters affecting the transformation of AgNPs were controlled during their preparation, storage, testing and applications. Anaerobic and basic conditions were maintained to avoid oxidation and dissolution, respectively. Ultrapure deionized water was used to avoid aggregation. The AgNPs were prepared and stored in the dark to avoid oxidation and were never subjected to vortex or sonication before use. During sensor fabrication, the AgNPs were reacted with the MMT linker immediately after their synthesis and stored until use at room temperature. The AgNPs after sulfidation (Ag-S) by reacting with MMT, is more stable in solution than are citrate-capped AgNPs (Ag$\mathrm{O})$. During toxicity testing the basic and anaerobic conditions were maintained by growing the yeast in alkaline media ( $\mathrm{pH}$ : 8-8.5) and in limited air. The coating of BSA and MAbs around AgNPs might be the reason for the decreased anti-microbial activity of the sensor compared to the bare AgNP substrate (10\% vs. 15\%). However, the equivalent dose of ionic silver was highly toxic (63\%). The results suggest that coating the AgNPs avoids their dissolution and release of ionic silver $\left(\mathrm{Ag}^{+}\right)$, which is far more toxic than the NPs themselves. Our results are in agreement with other groups that characterized the dissolution of $\mathrm{AgNPs}$ to $\mathrm{Ag}^{+}$as the main cause of toxicity $[36,37]$. The mixed culture was more resistant to the toxic effect of Ag than was the pure BY4742 strain of S.c. (Figure 3 right). The increased resistance of the mixed culture may be because it contains a number of wild and prototrophic strains of S.c. that possibly have evolved better metal homeostasis during their adaptation to harsh environmental conditions, including industrial effluents such as metals and chemicals. The strain-specific toxicity of AgNPs is reported elsewhere [38].

\section{Extracellular detection of stress-proteins by ELISA and SERS sensor}

The mixed culture grown under optimum conditions to log phase $\left(10^{7}\right.$ cells $\left./ \mathrm{ml}\right)$ was exposed to incremental doses of stress-toxins. The chemical-lysis method we used resulted in extraction of proteins with high purity (maximum absorption at $286 \mathrm{~nm}$ and $\mathrm{OD}_{280} / \mathrm{OD}_{260}=1.5$ ) and yield (60 $\mu \mathrm{g} / \mathrm{ml}$ culture), that is comparable to previous work $[39,40]$. The stress-markers, HSP70 and RAD54, expressed in response to stress-toxins, were specifically detected in the cell lysates using commercial ELISA kits, followed by their detection using the fabricated SERS sensors. The label-free SERS sensor shows good correlation in accuracy with the standard label-based ELISA technique for the extracellular detection of the dose dependent expression of HSP70 and RAD54 (Figure 4). A several fold increase in the levels of HSP70 (8x) and RAD54 $(4 \mathrm{x})$ was observed, which is in agreement with previous work [41,42]. The HSP70 (inductive isoform) measured shows very low baseline levels (almost undetectable) but high induction in response to stress [43] as compared to RAD54.

ELISA and SERS have comparable sensitivities (LLD 50 pg/ml) for the detection of HSP70 and RAD54. However, the reproducibility (standard deviation as well as correlation coefficient) of the ELISA was better than SERS (insets Figure 4). The reproducibility of the SERS sensor is particularly decreased with increasing concentration of the proteins. The poor detection range of the SERS, particularly at higher concentrations, has been reported [23]. SERS is an aggregationbased phenomenon that leads to an increase in optical scattering area. However, aggregation is a random process and hence controlling the hot spots that lead to the generation of the SERS signals is very difficult. The reproducibility ( $<20 \%$ deviation in SERS intensity) and sensitivity (LLD: $\leq 0.05 \mathrm{ng} / \mathrm{ml}$ and LRD: $0.05-2.5 \mathrm{ng} / \mathrm{ml}$ ) of our colloidal sensor to measure proteins in solution (insets Figure 4) are comparable to that achieved by the labor intensive, time-consuming and costly approach of patterning SERS substrate [21,22]. Compared to most of the other Raman instruments for SERS applications, the RamanStation $400 \mathrm{~F}$ offers high power at the sample that allows a very high signal/ noise ratio, and a larger spot size that allows analysis of a large area, and hence allows acquisition in mapping mode (SERS mapping). The acquisition of a SERS spectra using high power $(100 \mathrm{~mW})$ at the sample might be the reason for high sensitivity, and acquiring spectra in mapping mode instead of conventional point focus might have resulted in high reproducibility of our colloidal sensor. Our SERS sensor was comparatively more sensitive for the detection of RAD54 $(25 \mathrm{pg} / \mathrm{ml})$ than HSP70 $(50 \mathrm{pg} / \mathrm{ml})$. The increased sensitivity for the RAD54 detection is because of its higher Raman activity, possibly due to a stronger interaction with the sensor over HSP70. RAD54 is a larger protein structure than HSP70 (84 KD with 738 amino acids vs. 70 KD with 642 amino acids) with more repeated SERS active aromatic amino acids. Although both proteins are large molecules, they are
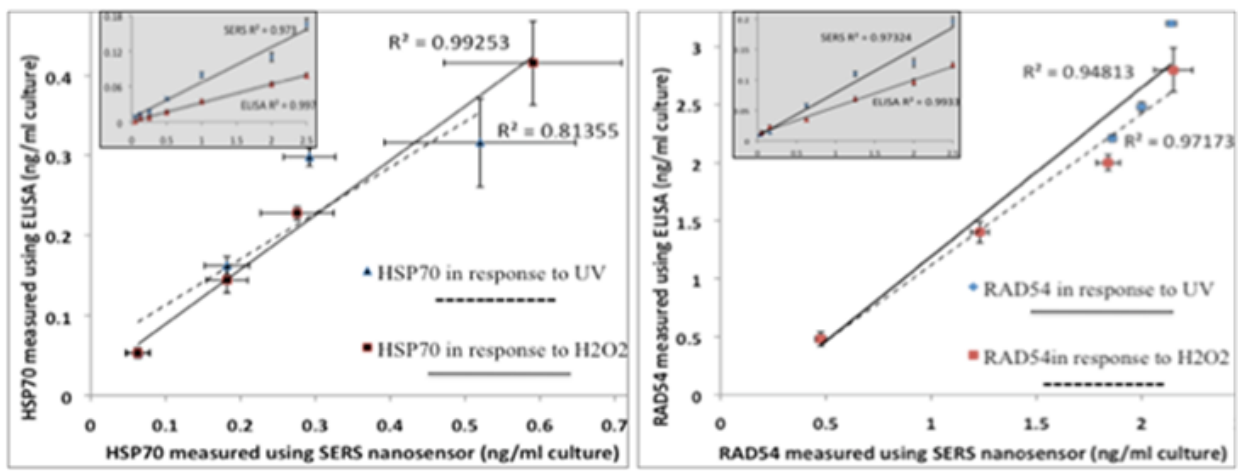

Figure 4: Correlation in accuracy of SERS with ELISA sensor for the extracellular detection of stress-proteins, HSP70 (left) and RAD54 (right). Both techniques show good correlation $\left(r^{2}\right)$ in the levels of stress-proteins measured after exposing yeast to incremental doses of toxins, $\mathrm{H}_{2} \mathrm{O}_{2}$ and $\mathrm{U}$.V. The inset shows the standard curves (amount in $\mathrm{ng} / \mathrm{ml}$ vs. intensity) used to compare the sensitivity of the two techniques, measuring the pure proteins in solution. SERS intensity $\left(\times 10^{6}\right)$ is measured at $1390 \mathrm{~cm}^{-1}$ and the ELISA intensity is measured at $450 \mathrm{~nm}$. $1 \mathrm{ml}$ culture $=10^{7}$ cells. The error-bars represent the S.D. of $n=3 \times 2$ (three independent experiments done in duplicates). 
Citation: Bhardwaj V, Srinivasan S, McGoron AJ (2013) AgNPs-Based Label-Free Colloidal SERS Nanosensor for the Rapid and Sensitive Detection of Stress-Proteins Expressed in Response to Environmental-Toxins. J Biosens Bioelectron S12: 005. doi: 10.4172/2155-6210.S12-005

Page 6 of 7

poor scatterers (i.e., they are not electron dense). Therefore, the SERS signals of these electron deficient biomolecules primarily depend on their adsorption or interaction with the AgNPs. The interaction and aggregation of proteins with the sensor depends on their surface charge (zeta potential) and the ionic species present in the suspension. As our study did not use any aggregating agent (ionic compound), the interaction is governed by surface charges on the protein and the sensor. The RAD54 protein has a higher isoelectric point (pI) than HSP70 (8.85 vs. 5.48) and hence shows higher affinity towards the negatively charged colloidal AgNPs ( $\mathrm{pH} 7-8$ ). The similar effect of charge on the interaction of proteins with the AgNPs has been reported previously [23].

Our SERS sensor is rapid and user-friendly as compared to ELISA. A typical commercial sandwich ELISA assay kit is supplied with a number of reagents (pre-coated wells, polyclonal and monoclonal Abs, Ab-conjugate, substrate and washing solutions etc.) that are to be used sequentially. This multi-step assay is labor-intensive and timeconsuming that takes at least 5-6 hours. The use of a series of peptides and the one-time use of wells make the kits fairly expensive. However, the protein detection using our colloidal SERS sensor is a two-step process that is simple to use and takes less than 2 hours to quantify the proteins. The samples (lysates) are incubated with the sensor for 90 minutes and the SERS signal measured using an automated Raman station 400F, that allows High Throughput Screening (HTS) in less than 5 minutes. Our sensor is more efficient than ELISA because it needs only the MAbs (single antigen-antibody binding event), no washing, no label and the wells are re-usable. Our sensor also has the potential for the real-time detection of proteins in whole-cells (i.e., to be a dynamic-sensor). Our label-free approach avoids any chances of uncertainty introduced by label-based sensors including ELISA, which indirectly determine the concentration of analyte by measuring signals from label-analyte conjugates. Besides quantification of the proteins, the SERS immuno-sensor also gives qualitative information about the interaction of the proteins (antigen and antibody), that is highly significant for immuno-sensor applications.

\section{Conclusion}

In our study, we demonstrated the application of SERS sensor technology for the extracellular detection of the stress-proteins HSP70 and RAD54 expressed by yeast in response to environmental toxins, U.V. and H2O2. In this report we also demonstrated the feasibility of replacing the standard ELISA technique by our sensor. SERS sensor has an edge over standard ELISA as it directly quantifies the proteins without using any label and also gives qualitative information about the assay (antigen-antibody interaction). The SERS outperforms ELISA in being rapid, user-friendly and low-cost. Both sensor techniques have comparable sensitivity. However, the SERS sensor is less reproducible than ELISA. The SERS sensor technique we developed offers: (i) a facile approach that uses colloidal AgNPs, (ii) high throughput screening of the proteins and (iii) can be used for intracellular detection as compared to previous work [21]. Bioreporter assays that use Genetically Modified Organisms (GMOs) for intracellular measurement of the stressinducible HSP70 [8] and RAD54 [9] in response to environmentaltoxins has been reported. However, owing to high incubation-time of the organism with the analyte, the assays cannot be used as a rapid "detect to protect" sensor device. Our SERS nanosensor allows rapid detection and has potential for intracellular monitoring of the stressproteins. Considering all these salient features of our sensor, it has the potential to be used for rapid monitoring of toxins in the environment, released deliberately (WAs) or accidentally (TICs). The multiplex and intracellular detection of the two proteins is underway with a goal to develop a yeast-based portable SERS sensor chip for monitoring environmental toxins.

\section{Acknowledgements}

We acknowledge the financial support from the Department of Defense U.S.A. (Award\# W81XWH-10-1-0732) for this study. The authors also acknowledge the Advanced Materials Engineering Research Institute (AMERI) at Florida International University for providing electron microscopy.

\section{References}

1. Sandhu P, Gupta D, Bhardwaj S, Jain S, Kataria S, et al. (2011) Enzyme-linked immuno-sorbent assay (ELISA), basics and it's application: A comprehensive review. J Pharmacy Res 4: 4581-4583.

2. Zadran S, Standley S, Wong K, Otiniano E, Amighi A, et al. (2012) Fluorescence resonance energy transfer (FRET)-based biosensors: visualizing cellular dynamics and bioenergetics. Appl Microbiol Biotechnol 96: 895-902.

3. van der Meer JR, Belkin S (2010) Where microbiology meets microengineering design and applications of reporter bacteria. Nat Rev Microbiol 8: 511-522.

4. Alford R, Simpson HM, Duberman J, Hill GC, Ogawa M, et al. (2009) Toxicity of organic fluorophores used in molecular imaging: literature review. Mol Imaging 8: 341-354.

5. Fang $\mathrm{Y}$ (2011) Label-free biosensors for cell biology. Int J Electrochem 2011 : 16.

6. Gooding JJ (2006) Biosensor technology for detecting biological warfare agents: Recent progress and future trends. Analytica Chimica Acta 559: 137 151

7. Harms H, Wells MC, van der Meer JR (2006) Whole-cell living biosensors-are they ready for environmental application? Appl Microbiol Biotechnol 70: 273-280.

8. La Terza A, Barchetta S, Buonanno F, Ballarini P, Miceli C (2008) The protozoan ciliate tetrahymena thermophilia as biosensor for sublethal levels of toxicants in the soil. FEB. 17: 1144-1150

9. Knight AW, Keenan PO, Goddard NJ, Fielden PR, Walmsley RM (2004) A yeast-based cytotoxicity and genotoxicity assay for environmental monitoring using novel portable instrumentation. J Environ Monit 6: 71-79.

10. Jeanmaire DL, Van Duyne RP (1977) Surface Raman spectroelectrochemistry Part I. heterocyclic, aromatic, and aliphatic amines on the anodized silve electrode. Electroan Chem Interf Electrochem 84: 1-20.

11. Culha M, Cullum B, Lavrik N, Klutse CK (2012) SERS as an emerging characterization and detection technique. J. Nanotech 2012: 15.

12. Culha M (2013) Surface-enhanced Raman scattering: an emerging label-free detection and identification technique for proteins. Appl Spectrosc 67: 355-364.

13. Baronian KH (2004) The use of yeast and moulds as sensing elements in biosensors. Biosens Bioelectron 19: 953-962.

14. Jamieson DJ (1998) Oxidative stress responses of the yeast Saccharomyces cerevisiae. Yeast 14: 1511-1527.

15. Notingher I, Green C, Dyer C, Perkins E, Hopkins N, et al. (2004) Discrimination between ricin and sulphur mustard toxicity in vitro using Raman spectroscopy. $J$ R Soc Interface 1: 79-90.

16. Lee PC, Meisel D (1982) Adsorption and surface-enhanced raman of dyes on silver and gold sols. J. Phys Chem 86: 3391-3395.

17. Levard C, Hotze EM, Lowry GV, Brown GE Jr (2012) Environmental transformations of silver nanoparticles: impact on stability and toxicity. Environ Sci Technol 46: 6900-6914

18. Payne EK, Rosi NL, Xue C, Mirkin CA (2005) Sacrificial biological templates for the formation of nanostructured metallic microshells. Angew Chem Int Ed Engl 44: 5064-5067.

19. Li H, Sun J, Cullum BM (2006) Label-free detection of proteins using SERSbased immuno-nanosensors. NanoBiotech 2:17-28.

20. Lee S, Lee J, Kim K, Sim SJ, Gu MB, et al. (2009) Eco-toxicity of commercial silver nanopowder to bacterial and yeast strains. Biotech Bioprocess Eng 14: 490-495.

21. Lee M, Lee S, Lee JH, Lim HW, Seong GH, et al. (2011) Highly reproducible 
Citation: Bhardwaj V, Srinivasan S, McGoron AJ (2013) AgNPs-Based Label-Free Colloidal SERS Nanosensor for the Rapid and Sensitive Detection of Stress-Proteins Expressed in Response to Environmental-Toxins. J Biosens Bioelectron S12: 005. doi: 10.4172/2155-6210.S12-005

immunoassay of cancer markers on a gold-patterned microarray chip using surface-enhanced Raman scattering imaging. Biosens Bioelectron 26: 21352141.

22. Malvaney SP, He L, Natan MJ, Keating CD(2003) Three-layer substrates for SERS: Preparation and preliminary evaluation. J. Raman Spect 34: 163-171.

23. Kahraman M, Sur I, Culha M (2010) Label-free detection of proteins from self-assembled protein-silver nanoparticle structures using surface-enhanced Raman scattering. Anal Chem 82: 7596-7602.

24. Emory SR, Nie S (1998) Screening and enrichment of metal nanoparticles with novel optical properties. J Phys Chem 102: 493-497.

25. Kerker M (1987) Estimation of SERS from surface-averaged electromagnetic intensities. J. Colloid Interf Sc 118: 417-421.

26. Lin XM, Cui Y, Xu YH, Ren B, Tian ZQ (2009) Surface-enhanced Raman spectroscopy: substrate-related issues. Anal Bioanal Chem 394: 1729-1745.

27. Vitol EA, Orynbayeva Z, Bouchard MJ, Azizkhan-Clifford J, Friedman G, et al. (2009) In situ intracellular spectroscopy with surface enhanced Raman spectroscopy (SERS)-enabled nanopipettes. ACS Nano 3: 3529-3536.

28. He L, Rodda T, Haynes CL, Deschaines T, Strother T, et al. (2011) Detection of a foreign protein in milk using surface-enhanced Raman spectroscopy coupled with antibody-modified silver dendrites. Anal Chem 83: 1510-1513.

29. Kora AJ, Beedu SR, Jayaraman A (2012) Size-controlled green synthesis of silver nanoparticles mediated by gum ghatti (Anogeissus latifolia) and its biological activity. Org Med Chem Lett 2:17.

30. Han XX, Jia HY, Wang YF, Lu ZC, Wang CX, et al. (2008) Analytical technique for label-free multi-protein detection based on Western blot and surfaceenhanced Raman scattering. Anal Chem 80: 2799-2804.

31. Tuma R (2005) Raman spectroscopy of proteins: From peptides to large assemblies. J. Raman Spect 36: 307-319.

32. Xiao Y, Isaacs SN (2012) Enzyme-linked immunosorbent assay (ELISA) and blocking with bovine serum albumin (BSA)--not all BSAs are alike. J Immunol Methods 384: 148-151.
33. Buchwalow I, Samoilova V, Boecker W, Tiemann M (2011) Non-specific binding of antibodies in immunohistochemistry: fallacies and facts. Sci Rep 1: 28.

34. Bondarenko O, Juganson K, Ivask A, Kasemets K, Mortimer M, et al. (2013) Toxicity of $\mathrm{Ag}, \mathrm{CuO}$ and $\mathrm{ZnO}$ nanoparticles to selected environmentally relevant test organisms and mammalian cells in vitro: a critical review. Arch Toxicol 87: 1181-1200.

35. Chithrani BD, Ghazani AA, Chan WC (2006) Determining the size and shape dependence of gold nanoparticle uptake into mammalian cells. Nano Lett 6 : 662-668.

36. Maurer-Jones MA, Mousavi MPS, Chen LD, Buhlmann P, Haynes CL (2013) Characterization of silver ion dissolution from silver nanoparticles using fluorous-phase ion-selective electrodes and assessment of resultant toxicity to shewanella oneidensis. Chem Sci 4: 2564-2572.

37. Xiu ZM, Zhang QB, Puppala HL, Colvin VL, Alvarez PJ (2012) Negligible particle-specific antibacterial activity of silver nanoparticles. Nano Lett 12 4271-4275.

38. Ruparelia JP, Chatterjee AK, Duttagupta SP, Mukherji S (2008) Strain specificity in antimicrobial activity of silver and copper nanoparticles. Acta Biomater 4: 707-716.

39. Schött EH, Holzer H (1974) Purification and some properties of tryptophan synthase inactivase II from yeast. Eur J Biochem 42: 61-66.

40. von der Haar T (2007) Optimized protein extraction for quantitative proteomics of yeasts. PLoS One 2: e1078.

41. Wang Y, Gibney PA, West JD, Morano KA (2012) The yeast Hsp70 Ssa1 is a sensor for activation of the heat shock response by thiol-reactive compounds. Mol Biol Cell 23: 3290-3298.

42. Cole GM, Schild D, Lovett ST, Mortimer RK (1987) Regulation of RAD54- and RAD52-lacZ gene fusions in Saccharomyces cerevisiae in response to DNA damage. Mol Cell Biol 7: 1078-1084.

43. Scott MA, Locke M, Buck LT (2003) Tissue-specific expression of inducible and constitutive Hsp70 isoforms in the western painted turtle. J Exp Biol 206: 303-311. 